\title{
APPLICATION OF AUTOMATED TRAJECTORY DESIGN FOR PILOT FLIGHT ASSESSMENT DURING ROUTE FLIGHTS
}

\author{
Tadas MASIULIONIS ${ }^{1}$, Darius MINIOTAS ${ }^{2}$, Darius RUDINSKAS ${ }^{3}$, \\ Ramūnas KIKUTIS ${ }^{4}$, Gabrielè MASIULIONIENE ${ }^{5}$
}

Vilnius Gediminas Technical University, Sauletekio al. 11 LT-10223 Vilnius, Lithuania

E-mails: ${ }^{1}$ tadas.masiulionis@vgtu.lt (correspondingauthor); ${ }^{2}$ darius.miniotas@vgtu.lt;

${ }^{3}$ darius.rudinskas@vgtu.lt; ${ }^{4}$ ramunas.kikutis@vgtu.lt; ${ }^{5}$ gabriele.masiulioniene@vgtu.lt

Received 10 August 2017; accepted 12 September 2017
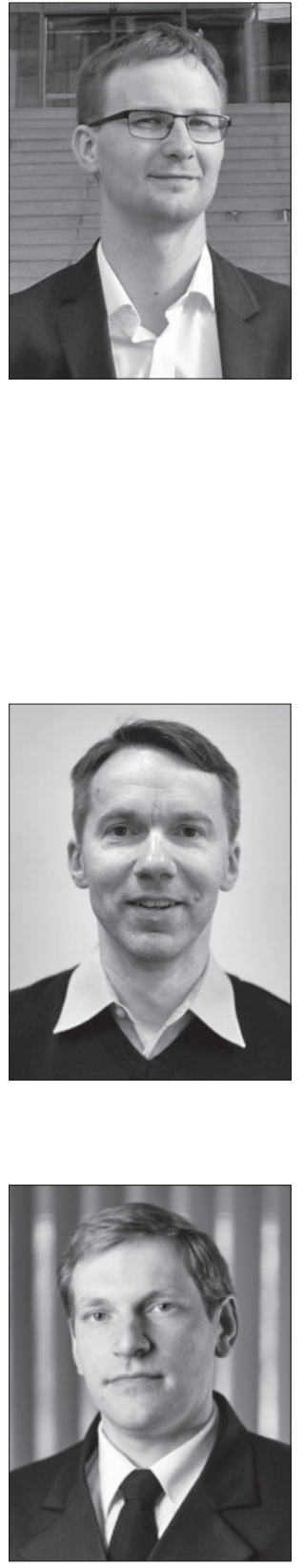

Tadas MASIULIONIS, $P h D$

Date and place of birth: 1984, Vilnius, Lithuania.

Education: 2017, Doctor of Transport Engineering, Antanas Gustaitis Aviation Institute, Vilnius Gediminas Technical University,; 2011, Master's degree, Antanas Gustaitis Aviation Institute, Vilnius Gediminas Technical University,.

Affiliations and Functions: since 2017, lecturer at the Department of Aeronautical engineering, Antanas Gustaitis Aviation Institute, Vilnius Gediminas Technical at University; 2015-2017, PhD student/lecturer at the Department of Avionics, Antanas Gustaitis Aviation Institute, Vilnius Gediminas Technical University; 2012-2015, PhD student/assistant lecturer, Department of Avionics, Antanas Gustaitis Aviation Institute, Vilnius Gediminas Technical University; 2010-2012, Aviation mechanic/avionic,at the Periodic maintenance department, FL Technics corp.

Research interests: pilot flight assessment.

Publications: author or co-author of 7 scientific articles, 6 conference papers.

Present position: lecturer at the Department of Aeronautical engineering, Antanas Gustaitis Aviation Institute, Vilnius Gediminas Technical University.

\section{Darius MINIOTAS, $P h D$}

Date and place of birth: 1970, Šiauliai, Lithuania.

Education: 2000, PhD, Kaunas University of Technology,; 1995, Master's degree, Kaunas University of Technology.

Affiliations and Functions:2014-2017, assoc. Professor at the Department of Avionics, Antanas Gustaitis Aviation Institute, Vilnius Gediminas Technical University; 2006-2014, assoc. professor at the Department of Electronic Systems, Vilnius Gediminas Technical University. Research interests: human-machine interaction, multimodal user interfaces. Publications: author or co-author of 36 scientific articles, 9 conference papers. Present position: lecturerat the Department of Electronic Systems, Vilnius Gediminas Technical University.

\section{Darius RUDINSKAS, $P h D$}

Date and place of birth: 1982, Rokiskis, Lithuania.

Education:2012, Measurement Engineering Doctor at Vilnius Gediminas Technical University, Antanas Gustaitis Aviation Institute; 2006, MSc in Electronic Engineering, Vilnius Gediminas Technical University, Faculty of Electronics; 2004, Electronic Engineer at Vilnius Gediminas Technical University, Faculty of Electronics.

Present position: lecturer and deputy director at Antanas Gustaitis Aviation Institute. Research interests: wireless sensors, integrated health management systems, flight control and navigation of UAVs. 


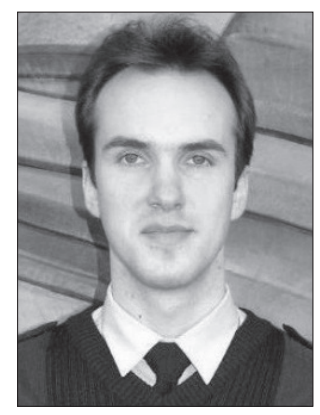

Ramūnas KIKUTIS, PhD student

Date and place of birth: 1988, Vilnius, Lithuania.

Education: Antanas Gustaitis Aviation Institute of Vilnius Gediminas Technical University, master degree of Transport engineering, 2012.

Affiliations and Functions: since 2013, PhD student at the Department of Avionics,

Antanas Gustaitis Aviation Institute, Vilnius Gediminas Technical University; since 2013, assistant lecturer at the Department of Aviation Technologies, Antanas Gustaitis Aviation Institute, Vilnius Gediminas Technical University; 2012-2013, researcher/assistant lecturer at the Department of Aviation Technologies, Antanas Gustaitis Aviation Institute, Vilnius Gediminas Technical University.

Research interests: autonomous UAV, flight accuracy, navigation.

Publications: author or co-author of 4 scientific articles.

Present position: assistant lecturer at the Department of Aviation Technologies, Antanas Gustaitis Aviation Institute, Vilnius Gediminas Technical University.

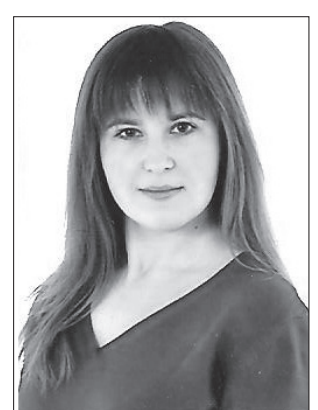

Gabrielè MASIULIONIENE், $M A$

Education: 2013, MA in English Studies, Vilnius University; 2011, BA in English Philology, Vilnius University.

Affiliation and functions: since 2013, assistant lecturer at the Department of Aviation Technologies, Antanas Gustaitis Aviation Institute, Vilnius Gediminas Technical University; since 2017, LR CAA approved English language proficiency examiner for pilots; since 2014, accredited examiner for ELPAC.

Research interests: aviation English, English for Specific Purposes, pilot training.

Abstract. Flight accuracy in the airspace is becoming an increasingly difficult issue due to the expanding number of aircraft operating in it. In order to meet the needs of all airspace users, aircraft flows are being increased, and different aircraft systems that minimise the risk of aircraft accidents are being developed to ensure flight safety. However, statistically, the impact of the human factor on aviation accidents and incidents remains high. This article focuses on the assessment of pilot flight accuracy during route flights and presents a methodology based on automated assessment tunnels for accurately assessing pilot flight deviations from a pre-set flight trajectory axis both on the horizontal and vertical plane.

Keywords: route flight, training flight assessment, automated flight accuracy assessment, flight assessment methodology, aviation.

\section{Introduction}

The use of the GNSS (Global navigation satellite system) in aviation has provided new possibilities for technological development in the area of air transportation. Most of the research on this topic is devoted to the improving satellite receiver positioning accuracy by applying various methods. It is now a common and even mandatory technical standard to purchase aircraft with an installed GNSS receiver (Jafernik et al. 2017). Therefore, it is not surprising that the number of pieces of equipment based on GNSS signals for calculating aircraft speed, time, and maintaining a defined position is increasing.

Other topics explored in current research are trajectory modelling (considering wind drift), flight optimization and forecasting, and flight accuracy improvement, which would help to improve the use of available airspace for an increasing aircraft flow (Liu, Luo 2011; Delahaye et al. 2014; Lee et al. 2015).

There are several research studies on the use of flight tunnels for pilot support systems (Nieuwenhuizen et al. 2014; Beringer 2000). However, tunnels used for the assessment of pilot flight accuracy have not been described. To fill this gap, in his article Masiulionis (2016) described the development and use of automated tunnel design for the assessment pilot flight accuracy in the traffic pattern, as well as issues that arise when using this method.

The aim of this paper is to explore the assessment pilot route flights by using automated trajectory design and to check the appropriateness of this method during route flight experiments.

\section{Computer model for the trajectory assessment of a route flight}

When pilots have shown that they are sufficiently experienced to carry out flights in the traffic pattern, they are permitted to fly route flights according to a filled flight plan. Usually, it includes the information about the pilot, the aircraft and the main data on the planned flight: the place and time of departure; flight altitude; 
speed and duration; flight route and destination. A flight route may be detailed by providing airport/aerodrome ICAO identification codes, names of towns, air navigation waypoints (region/upper information region), and all of these be defined by geographic coordinates. The flight trajectory can be plotted over some objects or waypoints. A pilot can complete the route at the initial point of departure, or land at any other destination.

Since the pilots can plan and design almost any configuration for the route flight trajectory, it is difficult for an instructor to assess the accuracy of a flight trajectory and the deviations from it. The application of automated flight trajectory design methods would permit more accurate assessments of a pilot's flight trajectory, independently of the selected route. Similarly to the method used in Masiulionis (2016), the route assessment tunnel has been designed with three levels of assessment, which correspond to the marks 10, 9 and 8 . If the pilot deviates from the set assessment tunnels, the flight is graded as a fail.

Straight line and arc elements were used by Shahzad et al. (2000) for modelling a flight trajectory. Figure 1 above illustrates the concept of such an assessment tunnel, using his calculations of the geometrical angles of the trajectory arcs. With this concept in mind, the design of the flight assessment trajectories during a route flight is based on initially set flight coordinates. The flight turn angles are calculated using trigonometric functions for three waypoint coordinates. Knowing the Cartesian coordinates of three waypoints, we can calculate the distances between the waypoints using Euclid's distance measuring equation between two points, which will allow calculating the route distance:

$$
D=\sqrt{\left(x_{1}-x_{2}\right)^{2}+\left(y_{1}-y_{2}\right)^{2}},
$$

where $D$ is the distance; $x_{1}, y_{1}$ - first waypoint coordinates; $x_{2}, y_{2}-$ second waypoint coordinates.

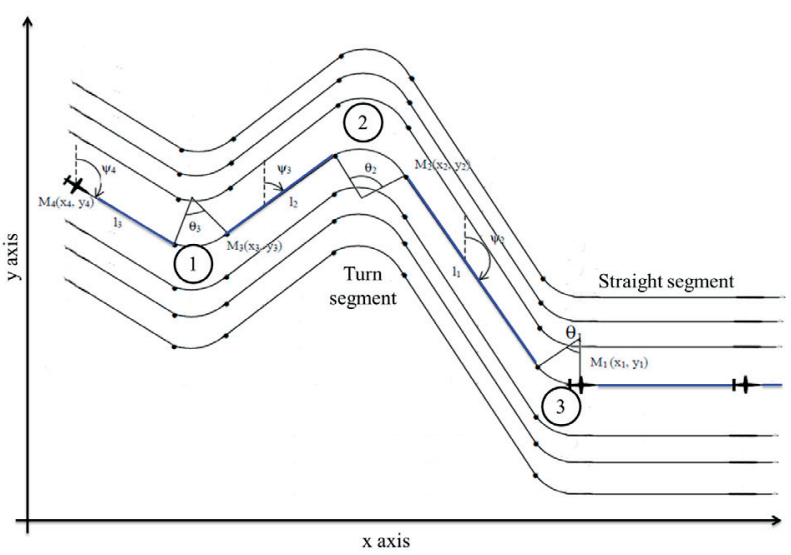

Fig. 1. Model design of the trajectory of a route flight using straight lines and arcs (Shahzad et al. 2000)
Afterwards, it is necessary to calculate the location of the centres of the turn arcs, define the turn radius and the widths of the assessment tunnels. However, during a route flight, it is difficult to determine the radius that is needed for turn arcs of turns with different turn angles, since, depending on the turn angle, the radius of the turn arc which would correspond to the real flight trajectory during the turn best changes.

Finally, it was decided to apply Bezier curves to design the route flight tunnel turns instead of the turn arcs. The turns are hence designed not according to the calculations of the arc center coordinates, but according to the known waypoint coordinates. For this, the initial and final coordinates of the Bezier curve, which are always connected to the straight lines between the other indicated waypoints, are calculated.

When forming the turn arc, it is necessary to indicate the distance which determines the sharpness of the appropriate route flight turn, along with an adequate turn arc for slower or faster flying planes. This distance is defined by ICAO as the DTA - distance to anticipation, and is shown in Figure 2 between the first and second waypoint. Based on this distance, the point where the pilot must start the turn to move from one straight flight segment to another is calculated. The same distance is also used between waypoints 2 and 3. According to the indicated distance, the coordinates of reference points 1 and 3 are calculated. When the three coordinates have been obtained, a Bezier curve can be plotted. Using the Matlab function for Bezier curve design provided by Narasimhan (2014), a $90^{\circ}$ aircraft flight turn according to the indicated waypoint coordinates 1, 2 and 3 has been drawn (Fig. 2).

In the following part, the methods for the design of a flight assessment tunnel for a route flight will be described in more detail. In the first stage of the route modelling for the flight assessment tunnel, we set the most important parameters for the tunnel: the tunnel is divided into three parts, with the width of each part

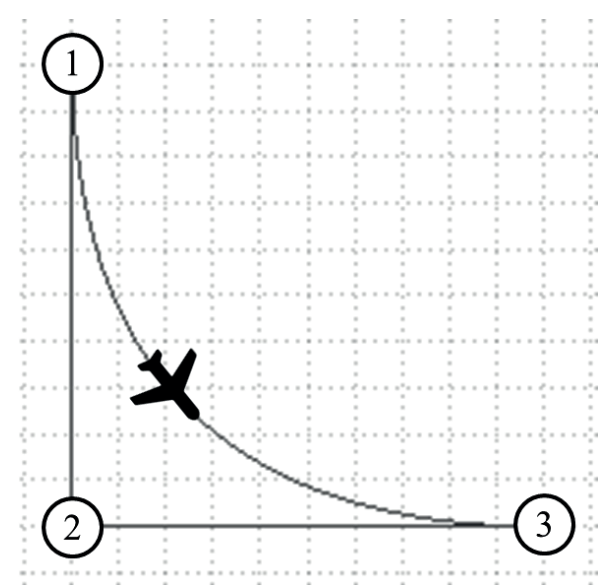

Fig. 2. Flight turn with a Bezier curve 
indicated, the DTA parameter is also defined; and the initially indicated waypoints are obtained from the CSV (comma separated values) file, that stores the flight's data for the route (names of waypoints and their latitude and longitude coordinates). Table 1 shows the data structure of the tunnel waypoints, obtained from the CSV, for the route flight assessment tunnel illustrated in Figure 3. Then, the number of the waypoints is determined, and the programme calculation cycles during which the geographical coordinates of the waypoints are transformed into Cartesian coordinates are repeated as many times. As a result, a graph of the coordinates is provided.

Table 1. Route flight data structure of waypoints in the CSV file

\begin{tabular}{lll}
\hline Waypoints & Latitude & Longitude \\
\hline Start & 54,66148 & 25,501968 \\
Rukainè & 54,599722 & 25,494444 \\
APSOL & 54,426389 & 25,327500 \\
Rūdiškès & 54,517018 & 24,824969 \\
Kurkliškès & 54,726723 & 24,845010 \\
KOTOV & 54,813610 & 25,056944 \\
Šaltoniškės & 54,869726 & 25,271632 \\
AukBez & 54,803797 & 25,592878 \\
Taurija & 54,737222 & 25,556111 \\
Finish & 54,671054 & 25,548349 \\
\hline
\end{tabular}

This procedure is followed by the calculation of angles. The turns of the route flight tunnels are made at different angles. In order to calculate the turn angles, the coordinates of three consecutive waypoints are selected, using the following Matlab function (2):

$$
W P_{\alpha}=\operatorname{atan} 2 \mathrm{~d}\left(\begin{array}{l}
\left.\operatorname{abs}\left(\operatorname{det}\left(\left[W P_{1}-W P_{2} ; W P_{3}-W P_{2}\right]\right)\right)\right), \\
\operatorname{dot}\left(W P_{1}-W P_{1}, W P_{3}-W P_{2}\right)
\end{array}\right),
$$

where $W P_{\alpha}$ is the angle of the turn between three waypoints in degrees (angles $\alpha_{1} \ldots \alpha_{8}$ calculated from Figure 3); $W P_{1}, W P_{2}, W P_{3}$ - waypoint coordinates expressed as $(x, y)$ (Fig. 3); atan2d - Matlab four-quadrant inverse tangent function with the result in degrees; abs Matlab function, expressing coordinate points in absolute numbers; det - Matlab function for the determinant; dot - Matlab function for scalar multiplication.

The flight assessment trajectory is modelled using straight lines and arcs, thus it is necessary to calculate the coordinates for intermediate points (Fig. 4), which would indicate where the lines and the arcs start. Similarly to the traffic pattern (Masiulionis 2016), the following circle equation is used for the calculations (3):

$$
\left\{\begin{array}{l}
x=R \cdot \cos (\beta)+x_{\mathrm{c}} \\
y=R \cdot \sin (\beta)+y_{\mathrm{c}}
\end{array}\right.
$$

where $x, y$ are the calculated circle perimeter point coordinates; $x_{c}, y_{c}$ - circle center coordinates; $R$ - circle radius; $\beta$ - a calculated angle which is at a right angle to the straight trajectory direction.

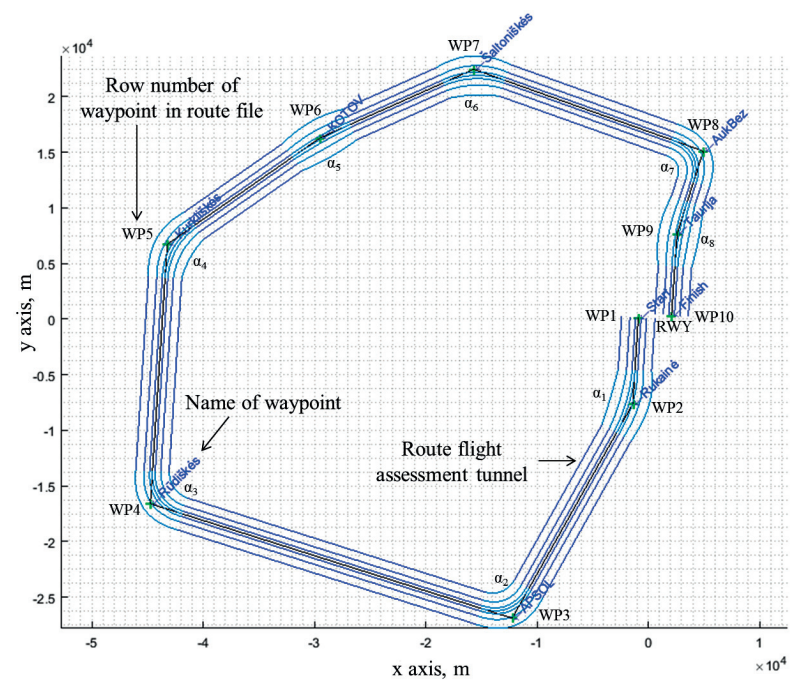

Fig. 3. Assessment tunnel model of route flight

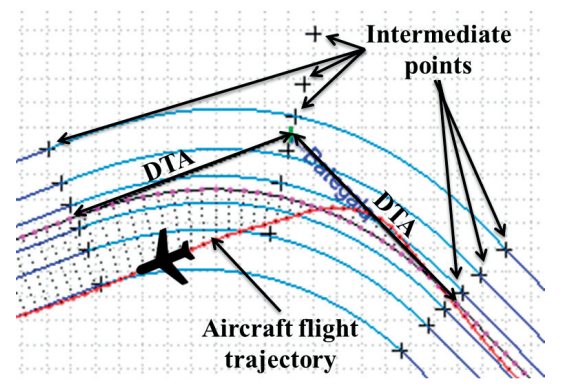

Fig. 4. Reference points of tunnel

Using the waypoint coordinates, equation (3) provides the coordinates for the new points, as well as the distance and the direction between waypoints. Both, backward (Fig. 2, 1<2) and forward $(2 \rightarrow 3)$ directions must be calculated. For these calculations, Matlab functions (4) and (5) respectively are used:

$$
\begin{aligned}
& c r s_{f}=\operatorname{atan} 2 \mathrm{~d}\left(y_{2}-y_{1}, x_{2}-x_{1}\right), \\
& c r s_{r}=\operatorname{atan} 2 \mathrm{~d}\left(y_{1}-y_{2}, x_{1}-x_{2}\right),
\end{aligned}
$$

where $c r s_{f}, c r s_{r}$ refer to the forward and backward directions in degrees; atan $2 \mathrm{~d}$ - Matlab four-quadrant arctangent function in degrees; $x_{1}, y_{1}-$ the coordinates of the first waypoint; $x_{2}, y_{2}$ - the coordinates of the second waypoint.

Apart from these calculations, it is also necessary to calculate the bisector (the direction of half an angle) using the Matlab function circcirc (an analogue is the Matlab function crossfix, which calculates the geographic coordinates of intersecting waypoints). The function calculates the intersecting points of two circles using the input coordinates for two waypoints and the lengths of their circle radius. In our scenario, both circles always intersect at two points; however, we cannot identify which coordinates refer to which intersection. To identify and 
link the coordinates with their respective intersections, a radius of both circles which is equal to the DTA distance is selected, so the centers of the circles correspond to the initial and the end points of the turn arc. With this solution, the coordinates of one of the intersections and one waypoint always coincide (Fig. 5). To identify the points, a conditional function which checks which intersecting point coincides with the waypoint is used. Additionally, Matlab function (4) is used to determine the direction of the bisector.

All reference coordinates that are calculated for the flight assessment are kept in a data matrix (Fig. 6). The odd lines of the matrix store $x$ coordinates (grey areas), and the even lines $-y$ coordinates (purple area). The reference coordinates of the levels of the flight assessment tunnel are stored in the columns. The first column corresponds to the reference coordinates of the inner tunnel (orange frame), the second - inner tunnel (green frame), and the third - outer tunnel (red frame).

If the matrix row has more columns, the sequencing of the data remains the same. The first four matrix lines store the reference coordinates for the first waypoint tunnel, which take up three columns. Lines 5 to 12 , store the coordinates for the tunnel's intermediate waypoints corresponding to the beginning and end of the Bezier

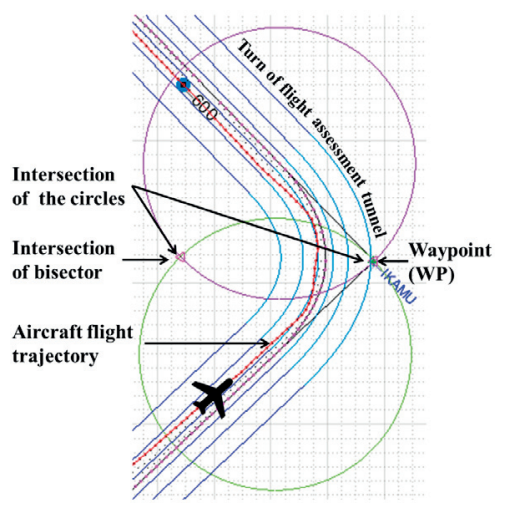

Fig. 5. Method for finding the turn bisector

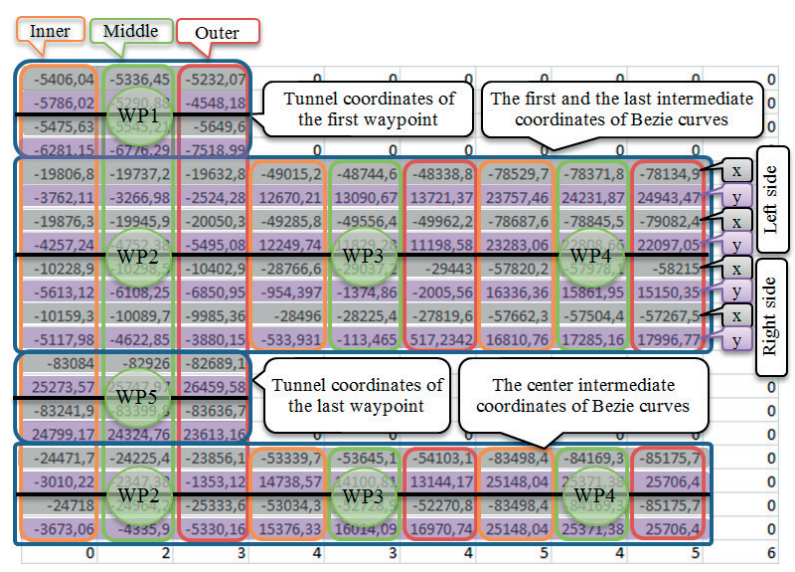

Fig. 6. Coordinate matrix for 5 waypoints of the route flight assessment tunnel curves. The number of columns in these rows depends on the number of waypoints used minus the first and last waypoint. The coordinates for the final point (rows 13-16) are formulated in the same way as for the first waypoint and also take up three columns. Rows 17-20 include the reference coordinates of the tunnel required for the inner Bezier curves.

In order to maintain the data sequencing, despite the zero which comes up in the last line of the matrix, an additional row (21) is included, in which the zero is stored but the line is not used. The other remaining zeros, not included in the frames, are also not used in the calculations. They are only necessary to even out the numbers of the matrix columns when carrying out the programme's calculation cycles.

To design turns, the direction in which the turn is executed must be determined (left or right) and the appropriate turn coordinates need to be selected accordingly. The turn direction is determined using three waypoints and the following Matlab functions (6) and (7):

$$
\begin{aligned}
& \text { crs }=\operatorname{cross}\left(\begin{array}{l}
{\left[x_{2}-x_{1}, y_{2}-y_{1}, 0\right],} \\
{\left[x_{3}-x_{1}, y_{3}-y_{1}, 0\right]}
\end{array}\right) ; \\
& \text { course }=\operatorname{sign}(\operatorname{crs}(3)),
\end{aligned}
$$

where cross is the Matlab function calculating a vector composed of two other vectors; $x_{1}, y_{1}$ - the first waypoint coordinate; $x_{2}, y_{2}$ - the second waypoint coordinate; $x_{3}, y_{3}$ - the third waypoint coordinate; sign - Matlab function recognizing the third element $\operatorname{crs}(3)$ result sign in the data (if $c r s>0$, then course $=+1$; if $c r s=0$, then course $=0$; if crs $<0$, then course $=-1$ ).

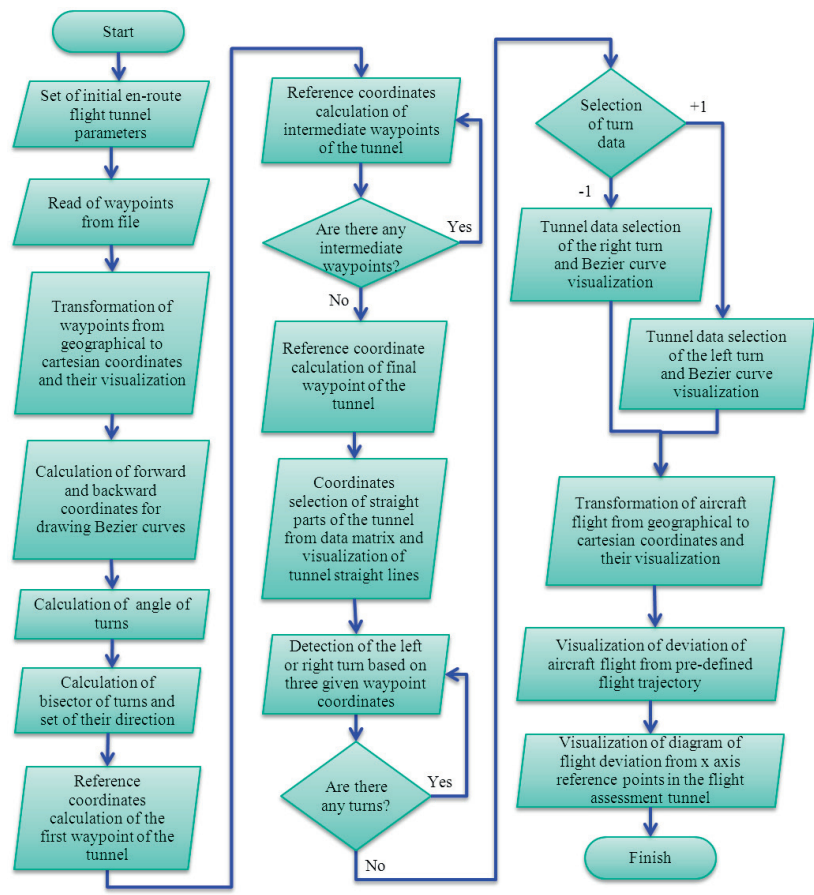

Fig. 7. Simplified algorithm of route flight modelling 
Afterwards, according to the conditions, the coordinates for the turn are chosen accordingly. If course $=+1$, then the coordinates for a left turn are selected; if course $=-1$ or 0 , then the coordinates for the right turn are selected. Finally, a complete model of an automatically designed tunnel for route flight assessment is obtained (Fig. 3). A simplified algorithm for the automated design of a route flight assessment tunnel is illustrated in Figure 7.

By using the automated design methods for the assessment tunnel described above and by uploading an airplane's flight trajectory into a computer, the instructor can easily design a tasks for any route flight, according to which, he can adequately assess a pilot's flight accuracy.

\section{Route flight experiment for model testing}

The aim of the experiment is to check the appropriateness of the tunnel for assessing the flight accuracy of real flights en-route.

A route flight is one of the final tasks for a pilot to improve his navigation skills. During this flight task, it is necessary for the pilot to maintain the trajectory set out in the flight plan accurately. Generally, pilots attempt to follow the waypoints outlined in an aeronautical chart. Therefore, to assess the trajectory of a pilot's flight objectively, we must take into account the waypoints that were selected by the pilot or the instructor.

Although the instructor also uses the aeronautical charts with the waypoints, it is difficult to make an accurate assessment. As a solution, an automated tunnel for route flight assessment has been designed to simplify the assessment of deviations form the planned flight trajectory. The deviations from the trajectory of a route flight can be spotted in both horizontal and vertical planes.

The curves of the assessment tunnel follow real flight trajectories. Having selected appropriate DTA distances, the aircraft pilot can easily, without any sharp

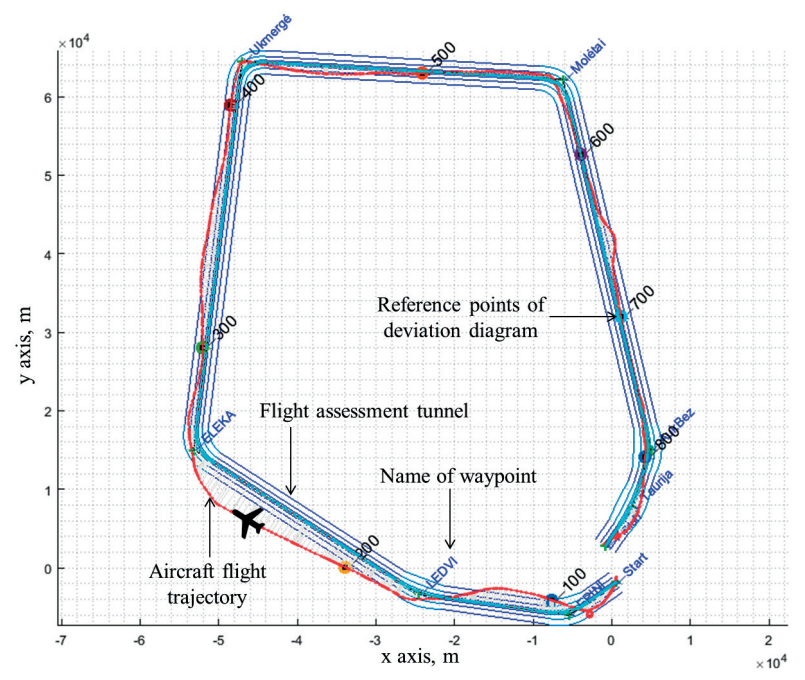

Fig. 8. Accuracy assessment of a route flight trajectory manoeuvres, stay inside of the tunnel. The tunnel may be designed for any flight route based on the selected waypoints, and the widths of the tunnel levels may be altered to take into consideration the pilot's experience.

The parameters of the flight assessment tunnel may be altered to meet instructor and training programme requirements; however, for the flight experiment (Figs 8 and 10), the waypoints have been pre-selected, and the same criteria for the assessment tunnels were used: a turn arc gradient (DTA) of $5000 \mathrm{~m}$, an assessment tunnel width of $1000 \mathrm{~m}$ for the inner, $2000 \mathrm{~m}$ for the middle, and $3000 \mathrm{~m}$ for the outer tunnel. Therefore, all pilots, despite the selected flight route, were assessed by using the same parameters for the assessment tunnels.

Similarly to the traffic pattern assessment (Masiulionis 2016), during the assessment of a route flight, a flight deviation measurement function, which measures airplane deviations from a set route and illustrates them graphically, is used. However, since we get one graph for the trajectory and the second one for the deviations, both with thousands of fixed GNSS points, it is difficult to compare the aircraft deviation and position parameters. To solve this, as seen in Figures 8 and 10, only the reference points of an aircraft's flight in the selected interval $(100,200, \ldots)$ obtained from the GNSS record are illustrated. The reference points correspond to the GNSS record points on the deviation graph's $x$ axis, and the deviation is expressed in positive numbers (Figs 9 and 11). Such a marking allows carrying out the inspection of the selected flight intervals more accurately. In order to determine inadmissible deviations automatically, you only need to concentrate on the deviation amplitude, which could alert the instructor by providing visual indications when the pilot exceeds the permitted values.

The horizontal lines in Figures 9 and 11 indicate the pre-set parameters of the assessment levels of the tunnel. The deviation graphs clearly indicate where the pilot did not meet the accuracy criteria and should repeat the flights to improve skills. Unfortunately, currently the designed model cannot take into account the meteorological conditions, so the pilot should have enough skills to

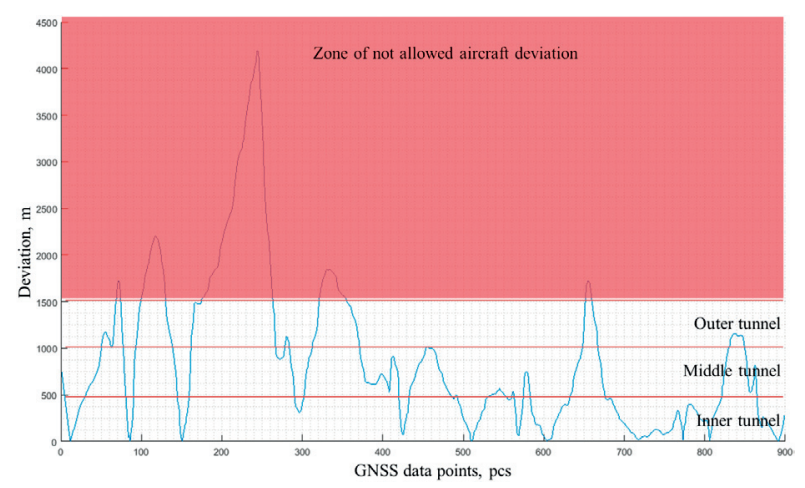

Fig. 9. Horizontal deviation of aircraft from predefined enroute flight from Figure 8 


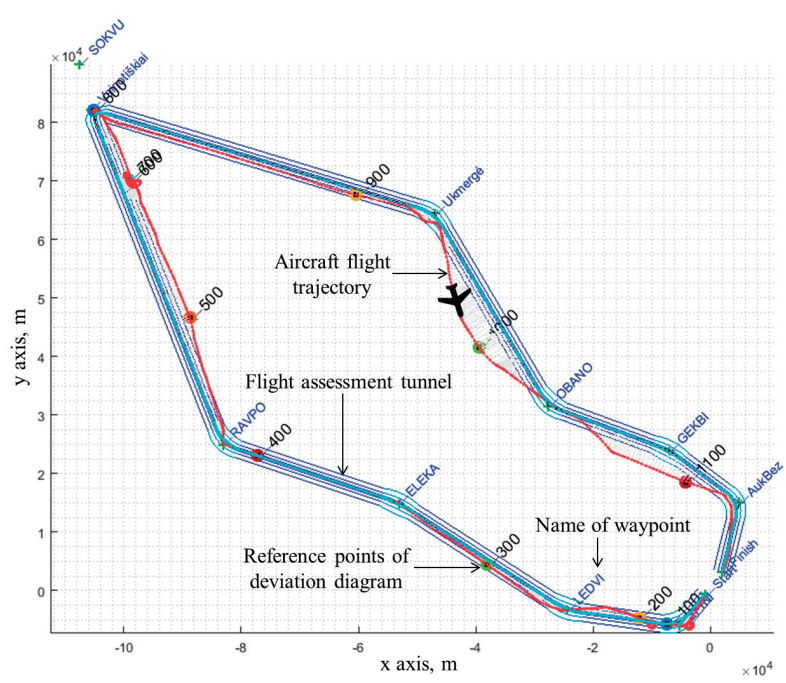

Fig. 10. Assessment model of a route flight

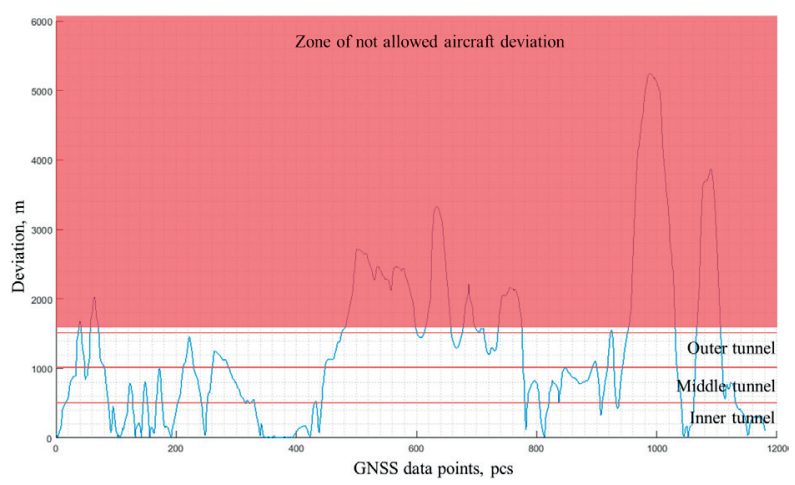

Fig. 11. Aircraft horizontal deviation from the route flight illustrated in Figure 10

be able to evaluate these conditions before his flight and to adjust his flying to meet the training requirements.

During the turns in a route flight, usually a fly-by technique is used. In this case, the pilot does not overfly a waypoint during the turn but gradually changes his/her direction from the initial course to the following course whenever he approaches a waypoint within a set DTA distance.

The comparison of the flight assessment tunnel with a real flight shows that the tunnel allows assessing flight deviations more precisely and also determining the places where the pilot deviated. The deviations are illustrated by a graph.

During a route flight, it is also important to monitor flight altitude. As seen in Figure 12, a different flight altitude can be specified between different waypoints, so, to carry out altitude assessment, the altitude levels should be changed automatically and inadmissible deviation parameter should be set again to permit automated alerts.

The position of an aircraft is determined using the Matlab inpolygon function. If specific areas are set

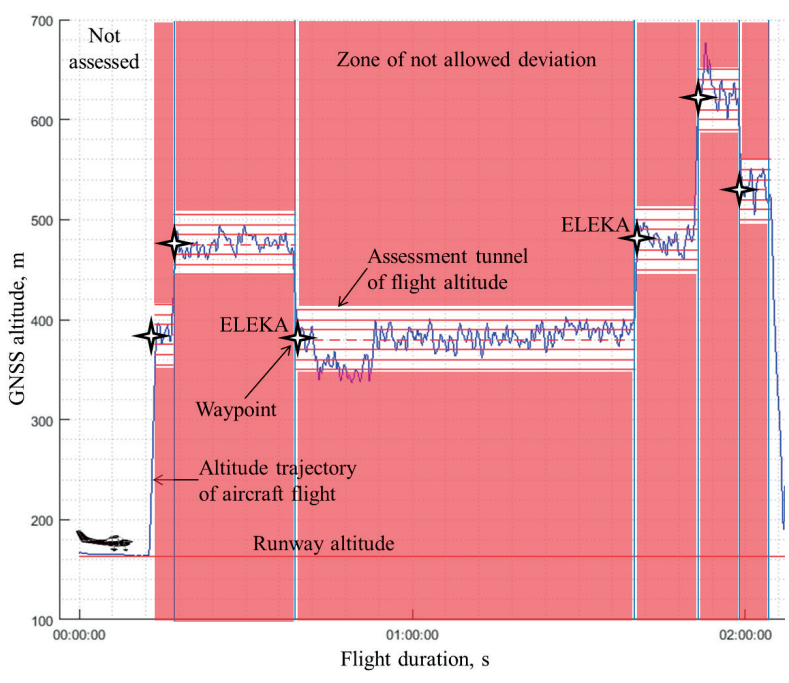

Fig. 12. Tunnel based airplane route flight altitude assessment

around each waypoint, when the pilot crosses them, we can automatically change the parameters for altitude assessment. Also, the flight interval should not be evaluated until the pilot has successfully reached the new altitude. The zone around each waypoint should be big enough for the pilot to be able to reach the required altitude. After measuring the distances needed to change the altitude, area distances of 2.6; 2.7; and $3.6 \mathrm{~km}$ were obtained (Fig. 12). To avoid inaccurate assessments, it is better to allow a greater margin. Therefore, the trigger zone width for altitude assessment should be at least $4 \mathrm{~km}$. The pilot's final mark will suffer when the pilot is not able to reach the required altitude in time.

When assessing the altitude in real flights, a $50 \mathrm{~m}$ tunnel height for maintaining flight altitude was found to be appropriate. According to the requirements of LR CAA, a private pilot is allowed to deviate by $\pm 30 \mathrm{~m}$.

An analysis of the altitude graphs of 20 pilot flights during which the pilots tried to maintain aircraft flight altitude showed that the standard deviation of flight altitude was between 3.80 and $28.06 \mathrm{~m}$. The standard deviation average square error has been calculated from the previously used trajectory altitudes and was equal to $15.65 \mathrm{~m}$., and the average was equal to $13.73 \mathrm{~m}$.

The tunnel model for route flight assessment can be improved in many ways by adding additional functions, which would allow the instructor to assess flight accuracy by selecting different flight parameters. For example, the flight tunnel model and the distance actually flown could be compared to find how fuel expenditure increased due to flight inaccuracy. Additionally, the ability to maintain aircraft speed and climb and descent rates could be assessed, as well as how long the pilot flew outside of the assessment tunnel. The pilot's en-route flight assessment model could also be improved by taking into consideration meteorological conditions. Using automated design 
methods for the flight assessment tunnel, a pilot's flight accuracy can be assessed both during and after the flight.

The developed flight assessment model can be applied for practical training of pilots and in aircraft flight accuracy competitions (airplane, helicopter, para-glider and UAV competitions), when there is a need to evaluate different flight trajectories.

\section{Conclusions}

1. It was found that to simplify the calculations for the design of the flight assessment tunnel model, the flight record coordinates have to be transformed from geographical (WGS-84) to local East North Up (ENU) coordinates.

2. The methods used for the modelling of a flight assessment tunnel must allow the model to freely adapt to changing turn angles.

3. The flight experiment showed that the developed methodology that uses several levels of assessment is appropriate for assessing the flight accuracy during route flights.

4. The analysis of flight altitude deviations during route flights revealed that, when maintaining the flight altitude, the standard deviation was between 3, 80 and 28, $06 \mathrm{~m}$. Thus, it did not exceed the limits on flight accuracy issued by the LR CAA. If more flight data were analysed, more precise flight accuracy requirements could be defined and categorised to meet specific training programmes.

5. The following solutions are required for the assessment methodology: an automated design of tunnels according to pre-set flight parameters, coordinate transformation of the aircraft flight trajectory, finding the turn direction, as well as calculating the angle and course between waypoints.

6. Flight accuracy assessment during the flight experiments showed that the assessment can be carried out for trajectories with different parameters. Objectivity is ensured by using flight assessment tunnels with the same parameters.
7. When comparing the data from the flight assessment tunnel and a real flight trajectory, the measurement equipment ensured a $\pm 5 \mathrm{~m}$ deviation form the real aircraft position.

\section{References}

Beringer, D. B. 2000. Development of Highway in the sky displays for flight path guidance: History, performance results, guidelines, Proceedings of the Human Factors and Ergonomics Society Annual Meeting 44(13): 21-24.

Delahaye, D.; Puechmorel, S.; Feron, E; Tsiotras, P. 2014. Mathematical models for aircraft trajectory design: a survey, Lecture Notes in Electrical Engineering 290 (Part V): 205-247.

Jafernik, H.; Fellner, A.; Mrozik, M.; Krasuski, K. 2017. Results of aircraft positioning tests in post-processing using the GNSS, Scientific Journal of Silesian University of Technology. Series Transport 95: 67-74. https://doi.org/10.20858/ sjsutst.2017.95.7.

Lee, A. G.; Wu, M. G.; Abramson, M. 2015. Modeling of complex and diverse aircraft trajectories with the trajectory synthesizer generalized profile interface, in AIAA Modeling and Simulation Technologies Conference, AIAA SciTech Forum, (AIAA 2015-0144), 5-9 January 2015, Kissimmee, Florida. https://doi.org/10.2514/6.2015-0144

Liu, C.; Luo, W. 2011. Probabilistic trajectory modeling of flight vehicles based on parameter deviations, in $2011 \mathrm{Sev}$ enth International Conference on Computational Intelligence and Security, 2011, Hainan, 468-471. https://doi.org/10.1109/CIS.2011.110

Masiulionis, T. 2016. Automatic assessment methods of a pilot's flight in the traffic circuit, Aviation 20(2): 53-59. https://doi.org/10.3846/16487788.2016.1195973

Narasimhan, L. 2014. Matlab code of generalised Bezier curve Matlab code, Mathworks [online], [cited $24 \mathrm{Au}-$ gust 2017]. Available from Internet: http://www.mathworks.com/matlabcentral/fileexchange/33828-generalised-bezier-curve-matlab-code

Nieuwenhuizen, F. M.; Heinrich, H.; Bülthoff, H. H. 2014. Evaluation of haptic shared control and a highway-in-thesky display for personal aerial vehicles, AIAA Modeling and Simulation Technologies Conference, AIAA SciTech Forum, (AIAA 2014-0808), 13-17 January 2014, National Harbor, Maryland.

Shahzad, M.; Mora-Camino, F.; Achaibou, K.; Slama, J. G. 2000. Trajectory generation and display for free flight, in ICAS 2000, 22nd Congress of International Council of the Aeronautical Sciences, Harrogates, UK. 